\title{
Intraductal Papillary Mucinous Neoplasms of the Pancreas
}

\author{
W.B. Al-Refaie E.A. Choi J.F. Tseng E.P.Tamm J.H. Lee J.E. Lee \\ D.B. Evans P.W.T. Pisters
}

Department of Surgical Oncology, The University of Texas M.D. Anderson Cancer Center, Houston, Tex., USA

\author{
Key Words \\ Intraductal papillary mucinous neoplasms · Pancreas • \\ Pancreatic neoplasms
}

\begin{abstract}
The introduction of the exocrine pancreatic classification by the World Health Organization and improvements in pancreatic imaging have led to an improved understanding of intraductal papillary mucinous neoplasms (IPMNs) of the pancreas. As a result, IPMNs of the pancreas are increasingly being recognized as a separate disease entity. IPMNs are characterized by the cystic dilatation of the pancreatic duct and its branches, with papillary projections. There are three histological subtypes of IPMNs: main duct, branch duct, and mixed. The degree of atypia ranges from adenoma to frank invasive carcinoma. The lymph nodes are involved considerably less frequently than they are in pancreatic adenocarcinoma. Most patients are symptomatic at diagnosis and require a diagnostic workup similar to that for patients with pancreatic adenocarcinoma. Although some investigators continue to advocate total pancreatectomy, the evidence in support of this is decreasing. Partial pancreatectomy remains the treatment option. Intraoperative assessment of the resection surgical margins is an important component of surgical resection. Additionally, controversy also exists regarding the nature of the follow-up
\end{abstract}

and the need for adjuvant chemoradiation therapy in the patient. Unlike ductal adenocarcinomas, IPMNs follow a relatively indolent course; the 5-year survival rate in patients with invasive IPMNs is $57 \%$. A mural nodule and a main pancreatic duct diameter greater than $5 \mathrm{~mm}$ have been found to be predictors of malignancy.

Copyright (C) 2006 S. Karger AG, Basel

\section{Introduction}

Owing to the most recent World Health Organization (WHO) classification [1] and progress in investigational techniques, there is a plethora of publications on multiple facets of intraductal papillary mucinous neoplasms (IPMNs) of the pancreas. This increased understanding of IPMNs of the pancreas has led to the recognition that this tumor is distinct from other cystic tumors of the pancreas. IPMNs are characterized by cystic dilatation of the pancreatic ducts, with papillary projections in the presence of excessive mucin production. These tumors account for less than $10 \%$ of all pancreatic neoplasms. Like other pancreatic tumors, surgical extirpation is the primary method of treatment. There is, however, debate regarding the extent of the pancreatic resection. Unlike pancreatic adenocarcinomas, IPMNs follow a relatively indolent course; the 5-year survival rate for patients with invasive IPMNs is up to $60 \%$ [2].

\section{KARGER}

Fax +4161306 1234

E-Mail karger@karger.ch

www.karger.com
(C) 2006 S. Karger AG, Basel

$1011-7571 / 06 / 0154-0245 \$ 23.50 / 0$

Accessible online at:

www.karger.com/mpp
Peter W.T. Pisters, MD

Department of Surgical Oncology, Unit 444

The University of Texas M.D. Anderson Cancer Center, 1515 Holcombe Boulevard Houston, TX 77230-1402 (USA)

Tel. +1 713794 1572,Fax +1 713792 7829, E-Mail ppisters@mdanderson.org 


\section{Historical Background}

IPMNs of the pancreas are described extensively in the Japanese literature. Ohashi et al. [3] first described IPMNs of the pancreas in 1981 in a report of 4 cases of mucinproducing cancers of the pancreas. IPMNs are also termed mucin-secreting carcinoma, villous adenoma of Wirsung's duct, diffuse intraductal papillary adenocarcinoma, intraductal cystadenoma, mucinous duct ectasia, and intraductal papillary mucinous tumors.

Increased recognition of IPMNs in the literature is explained by (a) the reclassification of exocrine tumors of the pancreas by WHO, (b) improvements in current pancreatic imaging techniques and therefore an improved understanding of IPMNs, and (c) better outcomes from pancreatectomy reported at high-volume centers. This latter has led to a higher rate of pancreatectomies.

\section{Demographics}

Patients with IPMNs of the pancreas present in the 6 th to 7 th decade of life and those with malignant IPMNs tend to be 6.4 years older than patients with adenomas or borderline lesions [2]. Despite the significant male predominance noted in early publications, current reports have described equal gender distribution [2, 4-7]. However, a recent publication of a large retrospective study from Asia showed a continued male predominance in this population, which raises the question of whether or not a geographic variation exists for IPMNs [8]. Interestingly, the median age of patients with IPMNs is two decades older than that of patients with chronic pancreatitis. IPMNs appear to demonstrate no racial predilection, as shown by several studies done in patients of different ethnic backgrounds [2, 4-9].

\section{Histopathological Features of IPMNs}

IPMNs encompass a wide spectrum of epithelial changes, ranging from adenoma to invasive adenocarcinoma, with borderline tumors and carcinoma in situ falling in between these two extremes [10]. As in patients with mucinous cystic neoplasms, the resected tumor must be extensively sampled by the pathologist to rule out cancer $[11,12]$.

There are three macroscopic subtypes of IPMN: main duct, branch duct, and mixed variant. In the Johns Hopkins Hospital experience [6], the branch-duct type was the most common (46\% of cases). Noninvasive IPMNs were in about $70 \%$ of branch-duct type. The main-duct type was the second most common type, accounting for about $28 \%$ of cases. Fifty percent of main-duct type tumors were noninvasive. The mixed-variant type was the least common type: (26\%); $60 \%$ of these cases were noninvasive. The distribution of invasive IPMNs among the three subtypes was as follows: branch duct 30\%, main duct $25 \%$, and mixed variant $25 \%$. On the other hand, in the series of Jang et al. [8], of 208 patients, more than 50\% showed the macroscopic appearance of main-duct type. Likewise, D'Angelica et al. [5] noted that $69 \%$ of patients with IPMNs had the main-duct type.

The WHO classification of IPMNs categorizes these further into three histological types: adenoma, borderline (including in situ) cancer, and invasive cancer. Sohn et al. [6] found that borderline IPMNs are more common $(62 \%)$ than the invasive type (38\%). However, D'Angelica et al. [5] noted that the incidence of the borderline and invasive IPMNs is 15 and $48 \%$, respectively. IPMN adenoma seems to be the least common histological subtype $(10 \%)$ in most series [6].

It is important to distinguish IPMNs from other mucinous cystic neoplasms. Unlike IPMNs, mucinous cystic neoplasms are characterized by 'ovarian-like stroma' in histologic appearance, female gender predilection and distal location in the pancreas. Unless a fistula has formed to the pancreatic duct, mucinous cystic neoplasms rarely communicate with the pancreatic duct, whereas IPMNs commonly communicate with the pancreatic duct.

\section{Molecular Features of IPMNs}

\section{K-ras Mutations and p53 Overexpression}

The K-ras mutation is seen in $70-100 \%$ of patients with pancreatic cancer, and the prevalence of the mutation correlates with the degree of dysplasia. For example, the K-ras mutation is found in $16.7 \%$ of normal epithelium samples of IPMN but in $57.1 \%$ of specimens of highgrade dysplasia, carcinoma in situ and malignant forms of IPMN are combined; 553 overexpression has shown the same pattern, with more invasive types of IPMNs showing a greater overexpression of p53 [13].

\section{Mucin Expression}

Mucins (MUC 1-7) are glycoproteins expressed in different types of pancreatic neoplasms. MUC 2 and MUC 5 are highly expressed proteins in invasive-type IPMNs. However, MUC 1 is expressed in ductal adenocarcinoma 
of the pancreas, but not in invasive IPMNs. Invasive IPMN patients with MUC5AC mRNA expression display better survival than those without MUC5AC mRNA expression [14].

\section{DPC4 Gene}

The DPC4 (deleted in pancreatic cancer 4) gene encodes a tumor suppressor protein that is relatively specific to pancreatic cancer and that is mutated in $45-55 \%$ of pancreatic adenocarcinomas. This mutation, resulting in the DPC4 inactivation, occurs due to one of two defects: a loss of heterozygosity (25\%) or homozygous deletion (35\%). On the other hand, in pancreatic adenocarcinomas, DPC4 is strongly expressed in all benign forms of IPMNs and in $84 \%$ of invasive IPMNs [7, 13, 15].

\section{Predictors of Malignancy}

Several studies have retrospectively analyzed clinical and pathological markers as predictors of malignancy [4, 5, 8]. Wiesenauer et al. [16] reviewed 64 consecutive cases with a pathological diagnosis of IPMN by analyzing 12 clinical symptoms and found that the onset of diabetes mellitus and jaundice were strongly associated with malignant IPMN. The study also showed that elevated serum alkaline phosphatase and glucose levels (as occur in diabetes) were strongly associated with invasive IPMN. Diabetes and jaundice were also found to be statistically significant, present in 140 patients with these tumors. Moreover, invasive adenocarcinoma was found in 60\% of patients with main-duct IPMNs. Several Japanese authors also searched for predictors of malignancy but made different findings. For example, in a series of 62 patients with IPMN, Sugiyama et al. [4] identified two independent predictors of carcinoma: mural nodules and a main pancreatic duct diameter of $7 \mathrm{~mm}$ or more. Similarly, Jang et al. [8] noted in their series of 208 patients that mural nodule $(\mathrm{p}=0.009)$, tumor diameter $\geq 3 \mathrm{~cm}(\mathrm{p}=$ $0.023)$, and dilated duct size $\geq 12 \mathrm{~mm}(\mathrm{p}=0.01)$ were predictors of malignancy. Although some predictors of malignancy have been reported consistently, the relatively small numbers of patients involved preclude analysis of their sensitivity and specificity. Hence, these proffer minimal indication in clinical decision-making.

There is a lack of consensus regarding pretreatment predictors for differentiating various types of malignant IPMNs. The ability to stratify patients into low- and highrisk categories would allow more objective, evidencedbased recommendations on resection treatment. More- over, in cases of partial pancreatic resections (pancreaticoduodenectomy or left pancreatectomy), an evaluation of other clinical, histological, and pathological markers would be helpful for follow-up and monitoring. Future research should focus on identifying clinicopathologic, radiologic and molecular factors that could be used for pretreatment risk assessment.

\section{Clinical Presentation}

Most reports suggest that more than $50 \%$ of patients with IPMNs are symptomatic [2, 5, 6], however, two thirds of the patients were asymptomatic at presentation in one study $[17,18]$. A minority of published studies suggest that a sizeable proportion of IPMN patients is asymptomatic at diagnosis, due, in part, to the recent improvement in radiological imaging of the pancreas $[6,8$, 19]. This fact, combined with the relatively indolent nature of some forms of the disease, creates challenges in individual patient management.

Unlike pancreatic ductal adenocarcinomas, 50\% of patients with IPMNs of the pancreas present with abdominal pain, and up to $20 \%$ of patients with IPMNs present with acute pancreatitis, which, not surprisingly, has led to the diagnosis of chronic pancreatitis $[2,4,6]$.

In patients with the invasive type of IPMN, weight loss and obstructive jaundice are the presenting features in 44 and $33 \%$ of the time, respectively [6]. However, patients with malignant IPMNs show a higher incidence of jaundice, recent onset or worsening of diabetes, longer duration of symptoms, and worsening abdominal pain compared to patients with benign IPMNs $[2,16]$. There might also be accompanying weight loss: $20-40 \%$; nausea and vomiting: $11-21 \%$; back pain: $10 \%$; steatorrhea: $6.5 \%$; gastrointestinal bleeding: $2-4 \%$ and fevers: $2-4 \%[2,5,6$, 16]. Although symptoms of jaundice or glucose intolerance might be more common, in patients with invasive forms of IPMN, the absence of these and other symptoms cannot be used reliably to exclude invasive IPMN.

IPMNs are distributed in the pancreas in the following proportions: head (50\%), tail (7\%), uncinate process (4\%), and neck of the gland (4\%). They may also occur throughout the gland in approximately $35 \%$ of cases [2]. 
Fig. 1. 46-year-old man with invasive IPMN identified on final histopathology. Contrast-enhanced preoperative baseline Multidetector CT image shows multiple cysts in the pancreatic head (white arrows) and soft tissue infiltrating into the pancreaticoduodenal groove (white arrowhead).

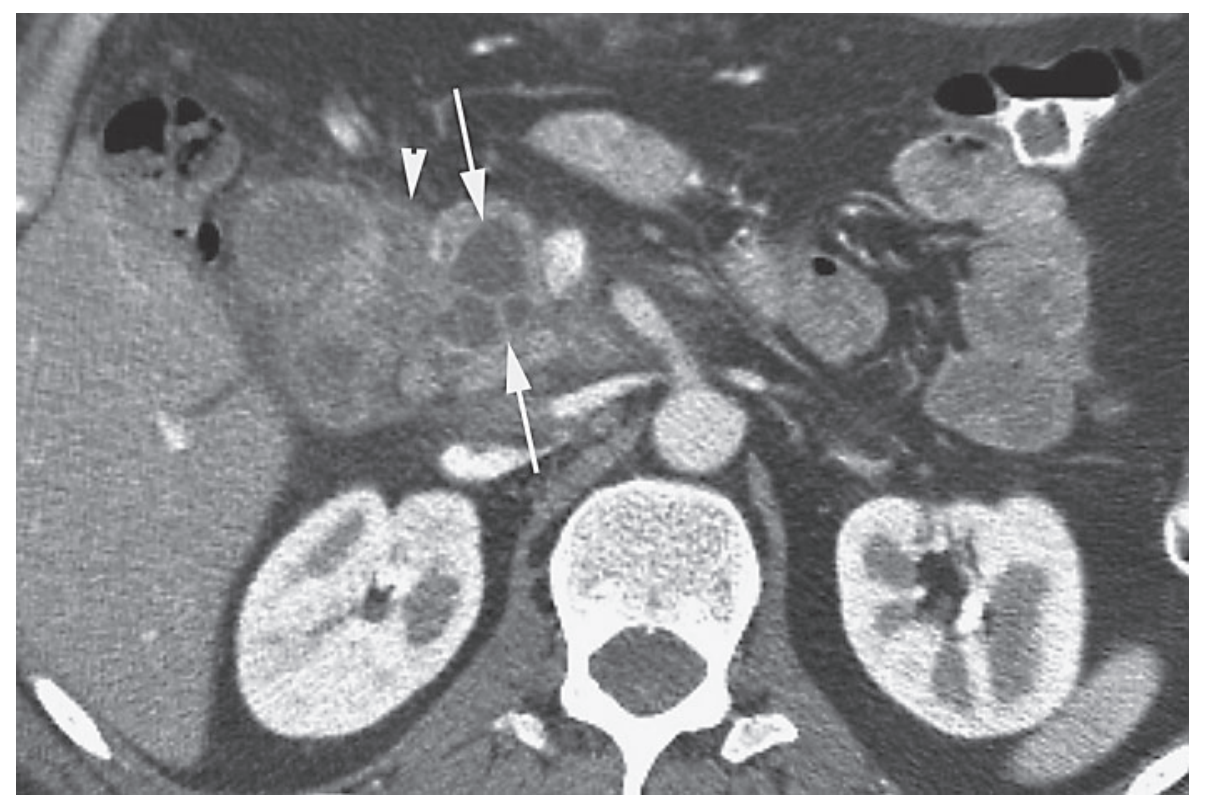

\section{Diagnostic Workup}

The diagnostic workup in patients with IPMNs of the pancreas is similar to that in patients with common pancreatic neoplasms. Following a thorough history and a physical examination, a complete blood count, electrolyte measurements, and a liver panel are performed. Since a proportion of patients present with a pancreatic mass, tumor markers, including CA 19-9, carcinoembryonic antigen, and CA 125 are also evaluated.

The primary imaging modality used to detect and evaluate IPMNs at the University of Texas M. D. Anderson Cancer Center is a thin-cut $(2.5-\mathrm{mm}$ sections, reconstructed to $1.25-\mathrm{mm}$ sections) dual-phase pancreatic protocol Multidetector CT scan of the abdomen and pelvis. In addition to delineating the nature and size of the mass and excluding hepatic and peritoneal metastasis, this allows the treating surgeon to evaluate the resectability and any extension of the tumor to other parts of the pancreas. The features of IPMNs shown by CT include a dilated main pancreatic duct, cysts of varying sizes, and possibly mural nodules (fig. 1). Multiplicity of cysts and associated downstream dilatation of the main pancreatic duct, when present, distinguish IPMNs from other cystic pancreatic neoplasms.

Pancreatography by endoscopic retrograde (ERCP) or magnetic resonance and endoscopic ultrasonography (EUS) are important secondary diagnostic tools for evaluation of patients with suspected IPMN. Pancreatogra- phy may allow for localization of mural nodules and pretreatment classification of suspected branch- or mainduct types of IPMN. During ERCP, the finding of viscous fluid oozing from a patulous ampulla of Vater is a classic endoscopic finding in patients with IPMN (fig. 2). EUS enables evaluation of pancreatic ducts and assessment of both the fluid and solid components within. Aspirated fluid is classically viscous, clear and contains mucin. $\mathrm{Cy}-$ tological studies demonstrate mucin-rich fluid with variable cellularity: columnar mucinous cells with variable atypia may also be seen.

Tumor markers are of variable utility, and there is no strong evidence in the literature that tumor markers in aspirated fluid are useful in determining the degree of malignancy of IPMNs. The levels of amylase and carcinoembryonic antigen may be high, but CA 19-9 and CA 125 levels may be low.

Intraoperative ultrasonography is of unclear utility in assessing these pancreatic lesions. However, intraoperative pancreatoscopy is used increasingly to inspect the ductal system of the remaining pancreas, as described below.

\section{Pancreatectomy for IPMN}

Partial pancreatectomy is the cornerstone treatment for main-duct and some symptomatic large $(>3 \mathrm{~cm})$ branch-duct IPMNs of the pancreas. However, the opti- 
Fig. 2. Viscous fluid oozing from a patulous ampulla of Vater in a patient with IPMN of the pancreas.

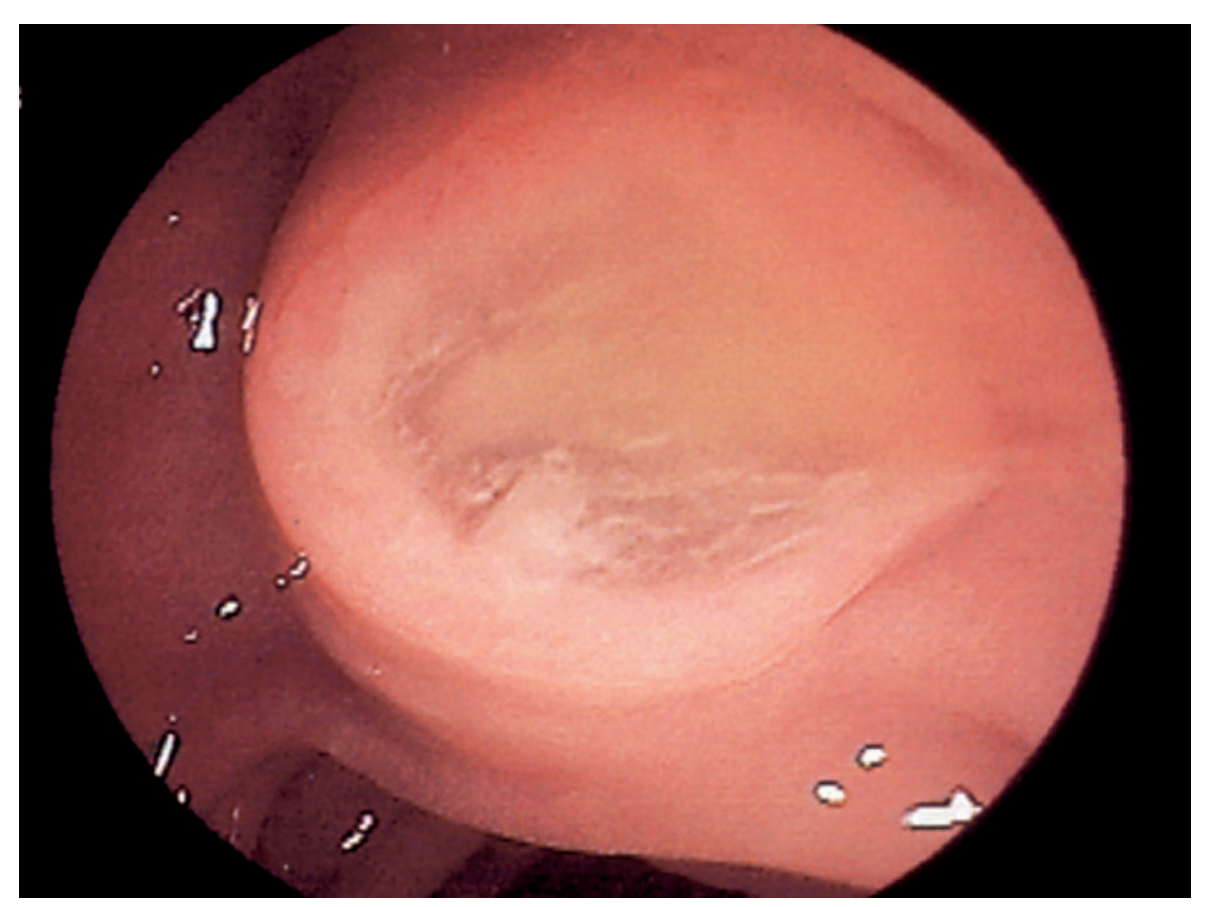

mal extent of pancreatic resection for some patients remains an open question. Most pancreatic surgeons recommend partial (usually right) pancreatectomy with the knowledge that the disease is most often located in the right side of the gland even though ductal changes may extend to involve other parts of the pancreas. Partial pancreatectomy also avoids the brittle diabetes that accompanies total pancreatectomy. Left (distal) pancreatectomy is the treatment of choice for the less common IPMNs that appear to be confined to the body and tail of the pancreas. Central pancreatectomy, or 'median pancreatectomy', has been performed by some for removal of localized tumors (likely branch-duct type) of the neck of the pancreas, but this has been associated with a higher incidence of pancreatic fistulas postoperatively and a higher tumor recurrence rate of $40 \%$ [20]. Total pancreatectomy is described for treatment of diffuse IPMNs of the pancreas. Although some investigators continue to advocate total pancreatectomy for the treatment of any IPMNs, evidence supporting this is decreasing with longer followup of patients treated by both R 0 and $\mathrm{R} 1$ partial pancreatectomy. Most surgeons recommend partial pancreatectomy but discuss the management of the pancreatic margin with the patient preoperatively and counsel that approximately $15 \%$ of patients will require conversion to total pancreatectomy in order to achieve negative parenchymal resection margins [2].
At most institutions, surgical margins are assessed intraoperatively, with additional margins obtained for carcinomas in situ. Intraoperative pancreatoscopy is currently utilized to inspect the ductal system of the remaining pancreas [21-24]. Macroscopic 'skip' lesions can be identified intraoperatively in the remnant pancreas by choledochoscopy. However, pancreatoscopy has some limitations: flat epithelium that looks normal on endoscopy can contain neoplastic changes. Unidentified occult multifocal disease has been proposed as the basis for recurrence of IPMNs in the remaining pancreas in patients who develop recurrent disease in the remnant pancreas, after macroscopic and microscopically complete (R0) resection of an ostensibly localized IPMN [6]. There is no evidence that any form of extended lymphadenectomy is indicated for patients undergoing pancreatectomy for IPMN or adenocarcinoma of the pancreas [24]. While the postoperative mortality rate varies from 0 to $5 \%$ at highvolume centers, overall postoperative complication rate following the resection of IPMNs is $30-50 \%$. Delayed gastric emptying and intra-abdominal sepsis are the most common complications [5,6]. The profile and frequency rates of complications in patients with IPMNs are not statistically different from those in patients with pancreatic ductal adenocarcinomas treated by pancreatectomy [6]. 
Table 1. Features and outcome of surgically treated IPMNs among various institutions

\begin{tabular}{|c|c|c|c|c|c|}
\hline Institution & Year & $\mathrm{n}$ & $\begin{array}{l}\text { Invasive } \\
\text { IPMN, \% }\end{array}$ & $\begin{array}{l}5-Y S^{a} \\
\%\end{array}$ & $\begin{array}{l}\mathrm{LN} \\
\%\end{array}$ \\
\hline Multi-institutional study (Korea) [8] & 2005 & 208 & 24.5 & 52.1 & 3.8 \\
\hline Virginia Mason Medical Center [26] & 2005 & 100 & 24 & 46 & 32 \\
\hline Johns Hopkins Hospital [6] & 2004 & 136 & 38 & 43 & 54 \\
\hline Massachusetts General Hospital and Verona Hospital, Italy [2] & 2004 & 140 & 41.6 & 60 & 41 \\
\hline Memorial Sloane Kettering Cancer Center [5] & 2004 & 63 & 48 & 58 & 16 \\
\hline Multi-institutional centers (France) [25] & 2002 & 73 & 70 & $67^{\mathrm{b}}$ & \\
\hline
\end{tabular}

LN = Lymph node involvement.

a 5-year survival for invasive-type IPMNs. ${ }^{\text {b }}$ 5-year survival for stage I invasive-type IPMNs.

\section{Outcome}

Survival rates are generally better in patients with IPMNs than in patients with pancreatic ductal adenocarcinoma (table 1) $[2,5,6,8,9,25,26]$. Sohn et al. [6] analyzed a series of 136 patients with IPMN and found that the survival rates for patients with noninvasive IPMNs were $97 \%$ at 1 year, $94 \%$ at 2 years, and $77 \%$ at 5 years. When the group of patients with noninvasive IPMNs was further analyzed, no differences were seen in survival rates between patients with IPMN adenoma and borderline IPMNs. Furthermore, this analysis failed to show any survival difference among all three IPMN types (mainduct, branch-type, or mixed-type). On the contrary, there was a significant survival difference between patients with noninvasive IPMNs and invasive IPMNs. The 1-, 3 -, and 5-year survival rates for patients with invasive IPMNs were 72,58 , and $43 \%$, respectively $(\mathrm{p}<0.0001)$. Therefore, survival appears to depend on the invasive component of the lesion.

Similar to the analysis by Sohn et al. [6], a study of 63 patients with IPMNs by D'Angelica et al. [5] reported disease-specific actuarial 5- and 10-year survival rates of 75 and $60 \%$, respectively. Furthermore, the survival rates in patients with noninvasive IPMN were better than those in patients with invasive IPMNs (5-year survival of 91 vs. $58 \%$, respectively, $\mathrm{p}<0.01$ ). Patients with tubulartype invasive IPMN (50\%) had a worse outcome than those with tubular colloid IPMNs $(73 \%, \mathrm{p}=0.008)$. Likewise, Jang et al. [8] found a significant difference in the 5-year survival rates in patients with adenomas: $93.5 \%$, borderline tumors: $91.4 \%$, noninvasive tumors: $84.7 \%$ and invasive tumors: $52 \%(\mathrm{p}=0.003)$. In addition, the 5 -year disease-free survival rates in patients with adeno- ma, borderline, noninvasive, and invasive IPMNs were $80.1,78.5,84.7$, and $36.9 \%$, respectively $(\mathrm{p}=0.001)$. These authors also noted a significant difference in survival between the groups of patients with benign (92.6\%) and malignant $(65.3 \%$ IPMNs, $p=0.006)$. However, no survival difference was seen among patients with mainduct (79.8\%), branch-duct (76.5\%), and combined-type $(80 \%)$ tumors $(p=0.728)$ [8]. On the other hand, Maire et al. [25] demonstrated in their multi-institutional French analysis of 73 IPMN patients that the overall 5-year survival in stage I invasive IPMN (67\%) was more favorable than that in a matched group of pancreatic ductal adenocarcinoma patients $(23 \%, \mathrm{p}<0.001)$. However, the authors showed that outcomes of locally advanced disease in both invasive-type IPMN and pancreatic ductal adenocarcinomas were similar [25]. Likewise, Wada et al. [26] reviewed their experience with 100 patients with IPMNs from Virginia Mason Center: a very high 5-year survival in patients with noninvasive IPMNs (100\%) was noted, compared to those with invasive IPMN $(46 \%, \mathrm{p}<0.001)$. When patients with invasive IPMNs $(\mathrm{n}=24)$ were matched (by TNM staging) to a similar group with pancreatic ductal adenocarcinoma $(n=24)$, the survival trend in favor of patients with invasive IPMNs was not insignificant $(p=0.110)$ [26].

IPMNs generally follow an indolent course. Although the small number of patients analyzed in reported studies and the short follow-up time preclude accuracy in determining the recurrence rate, in general, the recurrence rate is approximately $50 \%$ in patients with invasive IPMNs who have either negative or positive surgical margins and $8-10 \%$ in patients with noninvasive lesions. Furthermore, disease in patients with IPMNs with a focus of invasive carcinoma typically recurs in a disseminated form 
or at the pancreatic remnant. Noninvasive and originally benign IPMNs recur less often but can do so as an invasive adenocarcinoma locally or as disseminated disease. Thus, an $\mathrm{R} 0$ resection does not guarantee freedom from subsequent pancreatic remnant disease and, paradoxically, there are patients with noninvasive IPMN treated by R1 surgical resection who do not develop subsequent remnant recurrence.

As for pancreatic ductal carcinoma, pathological markers such as lymph node involvement, vascular invasion, and perineural invasion are associated with poor outcomes. That is, these findings are often seen in patients with aggressive, malignant tumors and locally advanced disease. Several studies have shown poor outcomes in patients with lymph node involvement. Although very few of their patients with IPMNs had a positive lymph node status and vascular and perineural invasion, D'Angelica et al. [5] demonstrated that positive lymph node status was associated with a poor outcome: the median survival in the 10 patients in their study with lymph node involvement was 29 months. Another study [6] revealed that, in patients with positive lymph nodes, the 5 -year actuarial survival rate was $0 \%$, compared to a rate of $85 \%$ in patients with negative lymph nodes.

It appears that local recurrence following pancreatectomy for invasive IPMNs may be high; however, this has not been extensively validated. In the series of Jang et al. [8], 36.1\% of patients who underwent R0 resections for invasive IPMNs developed locoregional failure: R0 resections for invasive IPMNs were performed in $83.7 \%$ of patients who underwent partial pancreatectomy for invasive IPMN, with disease recurring in $36.1 \%$ after partial pancreatectomy and in $37.5 \%$ after total pancreatectomy. Of those who underwent $\mathrm{R} 0$ resections for invasive IPMNs, disease recurred in $36.1 \%$, whereas disease recurred in only 1 patient out of the $7(14.2 \%)$ who underwent $\mathrm{R} 1$ resection [8]. One would expect that the locoregional failure rate would be higher among patients with R1 resections, but surprisingly, disease recurred more commonly in the liver. On the basis of these findings, the authors suggested considering postoperative systemic chemotherapy.

\section{Current Controversies}

Understandably, controversy still exists regarding the extent of pancreatectomy that should be performed in patients with pancreatic IPMNs. On the one hand, given the sometimes diffuse nature of IPMNs, total pancreatec- tomy would appear to be a logical choice, but on the other hand, total pancreatectomy is associated with a somewhat higher risk of postoperative complications and often results in brittle diabetes. Conversely, the favorable outcomes in patients with benign or borderline IPMNs who undergo partial pancreatectomy argue strongly against total pancreatectomy. Thus, in an effort to achieve a balance between competing goals of completeness of resection, and short- and long-term morbidity, most pancreatic surgeons recommend partial pancreatectomy followed by close surveillance.

It is also increasingly clear that not all patients with IPMN require operation. A recent consensus conference on guidelines has recommended observation for patients with small asymptomatic branch-duct IPMN $(<3 \mathrm{~cm})$ that has no associated nodularity [27]. A plan for watchful surveillance with delayed intervention in such patients is believed to be reasonable as the risks for malignancy with small, asymptomatic branch-duct tumors is low, most of the patients are elderly and the time required to develop invasive malignancy may be longer than their life expectancy [28, 29].

There is no defined role for adjuvant treatment of pancreatic IPMNs. The pattern of failure includes both local and distant (usually liver) sites and thus future studies of adjuvant chemotherapy and radiation treatment may be worthy of consideration.

In summary, although IPMNs of the pancreas are a separate group of pancreatic cystic tumors, the diagnostic workup is similar to that used for patients with more common pancreatic tumors. Partial pancreatectomy is still the standard of care for patients with main-duct IPMNs and symptomatic larger branch-duct disease. Older patients with asymptomatic, small branch-duct type IPMN without any ductal nodularity may be managed by careful monitoring and delayed intervention as needed. Patients with IPMNs have a more favorable outcome than patients with ductal adenocarcinomas of the pancreas. 


\section{References}

1 Kloppel G, Solcia E, Longnecker DS, Capella C, Sobin LH: Histological Typing of Tumours of the Exocrine Pancreas, 2nd ed. Berlin, Springer, 1998.

-2 Salvia R, Fernandez-del Castillo C, Bassi C, Thayer SP, Falconi M, Mantovani W, Pederzoli P, Warshaw AL: Main-duct intraductal papillary mucinous neoplasms of the pancreas: clinical predictors of malignancy and longterm survival following resection. Ann Surg 2004;239:678-685.

3 Ohashi K, Murakami Y, Maruyama M: Four cases of mucin-producing cancer of the pancreas on specific findings of the papilla of Vater (in Japanese). Prog Dig Endosc 1982;20:348351.

-4 Sugiyama M, Izumisato Y, Abe N, Masaki T, Mori T, Atomi Y: Predictive factors for malignancy in intraductal papillary-mucinous tumours of the pancreas. Br J Surg 2003;90: 1244-1249.

-5 D'Angelica M, Brennan MF, Suriawinata AA, Klimstra D, Conlon KC: Intraductal papillary mucinous neoplasms of the pancreas: an analysis of clinicopathologic features and outcome. Ann Surg 2004;239:400-408.

-6 Sohn TA, Yeo CJ, Cameron JL, Hruban RH, Fukushima N, Campbell KA, Lillemoe KD: Intraductal papillary mucinous neoplasms of the pancreas: an updated experience. Ann Surg 2004;239:788-797.

7 Sohn TA, Yeo CJ, Cameron JL, Iacobuzio-Donahue CA, Hruban RH, Lillemoe KD: Intraductal papillary mucinous neoplasms of the pancreas: an increasingly recognized clinicopathologic entity. Ann Surg 2001;234:313321.

-8 Jang J, Kim S, Ahn YJ, Yoon Y, Choi M, Lee K, Han J, Kim W, Lee YJ, Kim SC, Han DJ, Kim YI, Choi SH, Cho BH, Yu HC, Yoon DS, Lee WJ, Lee KB, Kim YC, Lee KS, Kim M, Kim HJ, Kim HJ, Park Y: Multicenter analysis of clinicopathologic features of Intraductal papillary mucinous tumors of the pancreas: is it possible to predict malignancy before surgery? Ann Surg Oncol 2005;12:124-132.

-9 Maire F, Couvelard A, Hammel P, Ponsot P, Palazzo L, Aubert A, Degott C, Dancour A, Felce-Dachez M, O'Toole D, Levy P, Ruszniewski P: Intraductal papillary mucinous tumors of the pancreas: the preoperative value of cytologic and histopathologic diagnosis. Gastrointest Endosc 2003;58:701-716.

10 Adsay NV: Intraductal papillary mucinous neoplasms of the pancreas: pathology and molecular genetics. J Gastrointest Surg 2002;6: 656-659.
-11 Adsay NV, Conlon KC, Zee SY, Brennan MF, Klimstra DS: Intraductal papillary-mucinous neoplasms of the pancreas: an analysis of in situ and invasive carcinomas in 28 patients. Cancer 2002;94:62-77.

12 Hruban RH, Takaori K, Klimstra DS, Adsay $\mathrm{NV}$, Albores-Saavedra J, Biankin AV, Biankin SA, Compton C, Fukushima N, Furukawa T, Goggins M, Kato Y, Kloppel G, Longnecker DS, Luttges J, Maitra A, Offerhaus GJ, Shimizu M, Yonezawa S: An illustrated consensus on the classification of pancreatic intraepithelial neoplasia and intraductal papillary mucinous neoplasms. Am J Surg Pathol 2004;28: 977-987.

13 Schmitz-Winnenthal FH, Z'Graggen K, Volk C, Schmied BM, Buchler MW: Intraductal papillary mucinous tumors of the pancreas. Curr Gastroenterol Rep 2003;5:133-140.

14 Yonezawa S, Horinouchi M, Osako M, Kubo M, Takao S, Arimura Y, Nagata K, Tanaka S, Sakoda K, Aikou T, Sato E: Gene expression of gastric type mucin (MUC5AC) in pancreatic tumors: its relationship with the biological behavior of the tumor. Pathol Int 1999;49:4554.

15 Wilentz RE, Iacobuzio-Donahue CA, Argani P, McCarthy DM, Parsons JL, Yeo CJ, Kern SE, Hruban RH: Loss of expression of Dpc4 in pancreatic intraepithelial neoplasia: evidence that DPC4 inactivation occurs late in neoplastic progression. Cancer Res 2000;60:20022006.

16 Wiesenauer CA, Schmidt CM, Cummings OW, Yiannoutsos CT, Howard TJ, Wiebke EA, Goulet RJ Jr, McHenry L, Sherman S, Lehman GA, Cramer H, Madura JA: Preoperative predictors of malignancy in pancreatic intraductal papillary mucinous neoplasms. Arch Surg 2003;138:610-617.

17 Tanaka M: Intraductal papillary mucinous neoplasm of the pancreas: diagnosis and treatment. Pancreas 2004;28:282-288.

18 Kawai M, Uchiyama K, Tani M, Onishi H, Kinoshita H, Ueno M, Hama T, Yamaue $\mathrm{H}$ : Clinicopathological features of malignant intraductal papillary mucinous tumors of the pancreas: the differential diagnosis from benign entities. Arch Surg 2004;139:188-192.

19 Conlon KC: Intraductal papillary mucinous tumors of the pancreas. J Clin Oncol 2005;23: 4518-4523.

20 Sauvanet A, Partensky C, Sastre B, Gigot JF, Fagniez PL, Tuech JJ, Millat B, Berdah S, Dousset B, Jaeck D, Le Treut YP, Letoublon C: Medial pancreatectomy: a multi-institutional retrospective study of 53 patients by the French Pancreas Club. Surgery 2002;132:836843.
21 Ozaki H, Kinoshita T, Kosuge T, Shimada K, Yamamoto J, Inoue K: Selection of methods for diagnosis and treatment of pancreatic cancer (in Japanese). Gan To Kagaku Ryoho 1992; 19:2311-2318.

22 Kanazumi N, Nakao A, Kaneko T, Takeda S, Harada A, Inoue S, Nagasaka T, Nakashima $\mathrm{N}$ : Surgical treatment of intraductal papillarymucinous tumors of the pancreas. Hepatogastroenterology 2001;48:967-971.

23 Tseng JF, Warshaw AL, Sahani DV, Lauwers GY, Rattner DW, Fernandez-del Castillo C: Serous cystadenoma of the pancreas: tumor growth rates and recommendations for treatment. Ann Surg 2005;242:413-419; discussion 419-421.

24 Pawlik TM, Abdalla EK, Barnett CC, Ahmad SA, Cleary KR, Vauthey JN, Lee JE, Evans DB, Pisters PW: Feasibility of a randomized trial of extended lymphadenectomy for pancreatic cancer. Arch Surg 2005;140:584-958; discussion 589-591.

25 Maire F, Hammel P, Terris B, Paye F, Scoazec JY, Cellier C, Barthet M, O'Toole D, Rufat P, Partensky C, Cuillerier E, Levy P, Belghiti J, Ruszniewski P: Prognosis of malignant intraductal papillary mucinous tumours of the pancreas after surgical resection: comparison with pancreatic ductal adenocarcinoma. Gut 2002; 51:717-722.

26 Wada K, Kozarek RA, William Traverso L: Outcomes following resection of invasive and noninvasive intraductal papillary mucinous neoplasms of the pancreas. Am J Surg 2005; 189:632-636.

27 Tanaka M CS, Adsay V, Fernandez-del Castillo C, Falconi M, Shimizu M, Yamaguchi K, Yamao K, Matsuno M: International Consensus Guidelines for Management of Intraductal Papillary Mucinous Neoplasms and Mucinous Cystic Neoplasms of the Pancreas. Pancreatology 2005;6:17-32.

28 Terris B, Ponsot P, Paye F, Hammel P, Sauvanet A, Molas G, Bernades P, Belghiti J, Ruszniewski P, Flejou JF: Intraductal papillary mucinous tumors of the pancreas confined to secondary ducts show less aggressive pathologic features as compared with those involving the main pancreatic duct. Am J Surg Pathol 2000;24:1372-1377.

29 Nakagohri T, Kenmochi T, Kainuma O, Tokoro Y, Asano T: Intraductal papillary mucinous tumors of the pancreas. Am J Surg 1999; 178:344-347. 